Pacific Journal of Mathematics

MARTINGALE PROOFS OF SOME GEOMETRICAL RESULTS 


\title{
MARTINGALE PROOFS OF SOME GEOMETRICAL RESULTS IN BANACH SPACE THEORY
}

\author{
Ken Kunen and HaSkell Rosenthal
}

\begin{abstract}
Martingale techniques are used to give a new proof of the theorem of J. Bourgain-R. R. Phelps that a closed bounded convex subset $K$ of a Banach space is the closed convex hull of its set of strongly exposed points provided $K$ has the Radon-Nikodym property. The new notions of " $\varepsilon$-strong extreme points" and the approximate Krein-Milman property are introduced, and the intimate connections between these notions and " $\delta$-trees" are explored. A self-contained treatment is given of the necessary martingale preliminaries, phrased in terms of quasi-martingales.
\end{abstract}

Our main objective is to render certain geometrical properties of convex sets accessible via the analytic techniques of martingale theory. For example, we give a new proof of the theorem of J. Bourgain [2] that a closed bounded convex subset $K$ of a Banach space is the closed convex hull of its set of strongly exposed points provided $K$ has the Radon-Nikodym property (the RNP). The result was first proved by $R$. $R$. Phelps assuming the entire Banach space has the RNP [9]. (Actually it has been observed by Larman and Phelps (see the remark following the proof of Theorem 4 of [8]) that a modification of Phelps' original argument can be used to obtain the above result of Bourgain). The arguments of Bourgain and Phelps seem to us to use ingenious but rather elaborate geometrical constructions. We obtain an essentially direct martingale proof via one geometrical result, Lemma 2.8. For the many equivalent formulations of the RNP, see [6]; we focus on the following one: $K$ has the RNP if and only if every $K$-valued martingale converges almost everywhere. We also introduce the new notion of " $\varepsilon$-strong extreme points" and the approximate Krein-Milman property, and show the intimate connections between this notion and " $\delta$-trees", that is, Banach valued dyadic martingales with differences everywhere $\delta$-bounded away from zero.

Let us now indicate in greater detail the organization of this work. In the first section, we develop the needed properties of a slight generalization of martingales, termed quasi-martingales in the literature. Most of our results here are special cases of known results of Bellow [1] and Edgar and Sucheston [7]. In Theorem 1.1 we show that every quasi-martingale may be decomposed as the sum of a martingale and a sequence tending to zero in $L^{1}$ norm 
and almost everywhere; (this is called the Riesz-decomposition in [7]). For the sake of completeness, we show in Theorem 1.3 that Banach valued $L^{1}$-convergent martingales converge almost everywhere. The final result of the first section is new; its proof shows the utility of quasi-martingales. This result, Proposition 1.6, implies that if $K$ is an RNP subset of a Banach space and $\left(f_{n}\right)$ is a uniformly bounded martingale "originating" in $K$, then if $\left(g_{n}\right)$ is the martingale obtained by stopping $\left(f_{n}\right)$ when it first exits $K,\left(g_{n}\right)$ converges almost everywhere.

The main results of the paper are contained in $\S 2$. We first give a proof in Proposition 2.1 of the standard result connecting dentability and the RNP. The argument reveals the power of the explicit use of quasi-martingales and also sets up techniques for later results.

We next treat the case of denting points. This case seems to illustrate the martingale techniques most intuitively, even though it is covered by the later one of strongly exposed points. (Phelps also treats denting points before strongly exposed points in his original paper [7].) The key geometrical result is given in Lemma 2.4. This result easily yields that an RNP set $K$ is the closed convex hull of its set of denting points (Theorem 2.2) and is "effectively" proved using quasi-martingales.

We treat the case of strongly exposed points in Theorem 2.5. After reviewing the natural, elegant geometrical preliminaries given by Bourgain in [2], we leave his treatment and present a new geometrical result, Lemma 2.8, which again is naturally accessible via martingales. The proof uses the new "stopping" result given at the end of the first section.

In the final section we introduce the new concept of a kind of approximate extreme point, called an " $\varepsilon$-strong extreme point"; and study the connection between its existence and $\delta$-trees; i.e., dyadic martingales with differences everywhere $\delta$-bounded away from zero. Precisely, if $K$ is a closed bounded convex set, $\varepsilon>0$ and $x \in K, x$ is called an $\varepsilon$-strong extreme point of $K$ if there is a $\delta>0$ so that if $k_{1}, k_{2} \in K$ satisfy $\left\|\left(k_{1}+k_{2}\right) / 2-x\right\|<\delta$, then $\left\|k_{1}-k_{2}\right\|<2 \varepsilon$. We show in Proposition 3.2 that if $K$ has no $\varepsilon$-strong extreme points, then $K$ contains $\delta$-trees for any $\delta<\varepsilon$. We also introduce the notion of $\varepsilon$-strong extreme points for sets $K$ which are not necessarily convex, and prove in Proposition 3.3 that a closed bounded convex set $K$ contains no generalized $\delta$-trees for any $\delta>0$ if and only if every nonempty subset of $K$ has an $\varepsilon$-strong extreme point for every $\varepsilon>0$. We do not know if these conditions imply that such a set $K$ is the closed convex hull of its $\varepsilon$-strong extreme points for every $\varepsilon>0$. However if this is not the case, we show in Proposi- 
tion 3.4 that $K$ admits a dyadic $K$-valued martingale with differences $\varepsilon$-bounded away from zero on a set of positive measure. There are known examples of Banach spaces $X$ failing the RNP yet containing no bounded $\delta$-trees [4]. Proposition 3.4 implies that for these known $X$ 's every closed bounded convex subset $K$ is indeed the closed convex hull of its set of $\varepsilon$-strong extreme points for every $\varepsilon>0$. It has also been independently observed by J. Bourgain and J. Elton that the $X$ 's of [4] fail the Krein-Milman property.

1. Quasi-martingales. We develop here the needed preliminaries from martingale theory. We employ a useful generalization of the concept of a martingale, something a bit more special than the "uniform amarts" introduced by A. Bellow in [1]. Our results here are also special cases of results of Bellow [1] and Edgar and Sucheston [7]; we present a self-contained treatment for the sake of completeness.

Let $X$ be a fixed Banach space and $(\Omega, \mathscr{S}, \mu)$ a fixed probability space. For a measurable function $f: \Omega \rightarrow X$ and $1 \leqq p \leqq \infty,\|f\|_{p}$ denotes the usual $L^{p}$ norm; $\|f\|_{p}=\left(\int\|f(\omega)\|^{p} d P(\omega)\right)^{1 / p}$ if $p<\infty$; $\|f\|_{\infty}=\operatorname{ess} \sup _{\omega \in \Omega}\|f(\omega)\| ; f \in L^{p}(X)$ if $\|f\|_{p}<\infty$. For $E$ a subset of $X$ and $f: \Omega \rightarrow X$ a given function, we denote $\{\omega \in \Omega: f(\omega) \in E\}$ by $[f \in E]$.

Suppose $\mathscr{A}$ is a $\sigma$-sub-algebra of $\mathscr{S}$ and $\mathscr{E}=\mathscr{E} \mathscr{A}$ denotes conditional expectation with respect to $\mathscr{A}$. That is, if $f \in L^{1}(X)$, then $\& f$ denotes the (essentially) unique $\mathscr{A}$-measurable integrable function so that

$$
\int_{A} \mathscr{E} f d P=\int_{A} f d P \text { for all } A \in \mathscr{A}
$$

We freely use the fact that then $\|\mathscr{E} f\|_{p} \leqq\|f\|_{p}$ for all $1 \leqq p \leqq \infty$. (Of course it is a rather nontrivial fact that there is a function " $\mathscr{f} f$ " with these properties. However we shall only employ the result for finite algebras $\mathscr{A}$, where this is obvious; in fact then for every atom $A$ of $\mathscr{A}, \mathscr{E} f \mid A$ is simply the constant given by $(1 / P(A)) \int_{A} f d P$.)

Now fix $\sigma$-sub-algebras $\mathscr{A}_{0} \subset \mathscr{A}_{1} \subset \mathscr{A}_{2} \cdots \mathscr{A}_{n} \subset \cdots$ of $\mathscr{S}$ with $\mathscr{A}_{0}$ the trivial algebra. Let $\mathscr{E}_{n}$ denote $\mathscr{E}_{\mathscr{N}_{n}}$, conditional expectation with respect to the $n$th algebra.

Definition. Let $1 \leqq p \leqq \infty$. A sequence $\left(f_{n}\right)$ of members of $L^{p}(X)$ is called a p-quasi-martingale (with respect to $\left(\mathscr{A}_{n}\right)$ ) if it satisfies the following two properties:

(a) $f_{n}$ is $\mathscr{A}_{n}$-measurable for all $n$, and 
(b) $\sum_{n=0}^{\infty}\left\|\mathscr{E}_{n} f_{n+1}-f_{n}\right\|_{p}<\infty$.

Let $\left(\varepsilon_{n}\right)_{n=0}$ be a summable sequence of nonnegative numbers. We say that the p-quasi-martingale $\left(f_{n}\right)$ corresponds to $\left(\varepsilon_{n}\right)$ if $\| \mathscr{E}_{n} f_{n+1}-$ $f_{n} \|_{p} \leqq \varepsilon_{n}$ for $n=0,1,2, \cdots$. We shall refer to 1-quasi-martingales as quasi-martingales. Of course a martingale $\left(f_{n}\right)$ with respect to $\left(\mathscr{A}_{j}\right)$ is simply a quasi-martingale corresponding to $\left(\varepsilon_{n}\right)$ where $\varepsilon_{n}=0$ for $n=0,1,2, \cdots$.

Our basic structural result is essentially contained in [1] and [7].

THEOREM 1.1. Let $1 \leqq p \leqq \infty,\left(\varepsilon_{n}\right)$ a summable sequence of nonnegative numbers, and $\left(f_{n}\right)$ a p-quasi-martingale (with respect to $\left.\left(\mathscr{A}_{n}\right)\right)$ corresponding to $\left(\varepsilon_{n}\right)$. Let $K$ be a closed convex subset of $X$ so that $f_{n}(\omega) \in K$ for all $n$ and $\omega$. Let $\delta_{n}=\sum_{j=n}^{\infty} \varepsilon_{j}$ for all $n$. Then there exists a martingale $\left(g_{n}\right)$ (with respect to $\left(\mathscr{A}_{n}\right)$ ) with the following properties for all $n$ :

and

(a) $g_{n}(\omega) \in K$ for all $\omega$

(b) $\left\|f_{n}-g_{n}\right\|_{p} \leqq \delta_{n}$.

Moreover $f_{n}-g_{n} \rightarrow 0$ a.e.

REMARK. It follows immediately that $\left|\int f_{n} d P-f_{0}\right| \leqq \delta_{n}+\delta_{0}$ for all $n$.

Theorem 1.1 yields the Riesz-decomposition for quasi-martingales, a special case of the decomposition given by Edgar and Sucheston in [7]. (The final assertion of 1.1 is established in [1].)

Before passing to the proof, let us note the following useful consequence (cf. [6]).

Corollary 1.2. Let $K$ be a closed bounded convex subset of $X$. Then the following are equivalent:

(a) $K$ has the RNP.

(b) $\left(f_{n}\right)$ converges almost everywhere for every quasi-martingale $\left(f_{n}\right)$ valued in $K$.

(c) $\left(f_{n}\right)$ converges in $L^{1}$-norm for every quasi-martingale $\left(f_{n}\right)$ valued in $K$.

Proof. We use the fact that this is true if we delete the word "quasi" in the statement. It thus suffices simply to prove that $(\mathrm{a}) \Rightarrow(\mathrm{b})$ and $(\mathrm{a}) \Rightarrow(\mathrm{c})$. Let $K$ have the RNP and $\left(f_{n}\right)$ be a quasimartingale valued in $K$. Let $\left(g_{n}\right)$ be the martingale given by Theorem 1.1. By $(a),\left(g_{n}\right)$ is valued in $K$. Thus since $\left(g_{n}\right)$ converges 
almost everywhere and in $L^{1}$-norm and $f_{n}-g_{n} \rightarrow 0$ almost everywhere and in $L^{1}$-norm by Theorem 1.1, $\left(f_{n}\right)$ converges almost everywhere and in $L^{1}$-norm.

We pass now to the proof of Theorem 1.1. We shall produce the martingale $\left(g_{n}\right)$ and prove that it satisfies (a) and (b). We then give some of the standard martingale results, cast in the language of quasi-martingales, to obtain that $f_{n}-g_{n} \rightarrow 0$ a.e.

We shall show, fixing $k$, that $\left(\mathscr{E}_{k} f_{n}\right)_{n=0}^{\infty}$ is Cauchy in $L^{p}$; then $\mathscr{E}_{k} f_{n}$ converges to a function $g_{k}$ in $L^{p}(X)$, as $n$ tends to infinity. In fact, fixing $n>k$, we have

$$
\left\|\mathscr{E}_{k} f_{n}-f_{k}\right\|_{p} \leqq \varepsilon_{k}+\cdots+\varepsilon_{n-1} .
$$

Indeed, $\mathscr{E}_{k} f_{n}-f_{k}=\mathscr{E}_{k}\left(f_{n}-f_{n-1}\right)+\mathscr{E}_{k}\left(f_{n-1}-f_{n-2}\right)+\cdots+\mathscr{E}_{k}\left(f_{k+1}-f_{k}\right)$.

But for $k+1 \leqq j \leqq n$,

$$
\mathscr{E}_{k}\left(f_{j}-f_{j-1}\right)=\mathscr{E}_{k}\left[\mathscr{E}_{j-1}\left[f_{j}-f_{j-1}\right]\right]=\mathscr{E}_{k}\left(\mathscr{E}_{j-1} f_{j}-f_{j-1}\right) .
$$

Hence $\left\|\mathscr{E}_{k}\left(f_{j}-f_{j-1}\right)\right\|_{p} \leqq\left\|\mathscr{E}_{j-1} f_{j}-f_{j-1}\right\|_{p} \leqq \varepsilon_{j-1}$, so $\| \sum_{j=k+1}^{n} \mathscr{E}_{k}\left(f_{j}-\right.$ $\left.f_{j-1}\right)\left\|_{p} \leqq \sum_{j=k+1}^{n}\right\| \mathscr{E}_{k}\left(f_{j}-f_{j-1}\right) \|_{p} \leqq \varepsilon_{k}+\cdots+\varepsilon_{n-1}$, proving (1). Since $L^{p}(X)$ is a Banach space, it follows that $\mathscr{E}_{k}\left(f_{n}\right)$ converges to an - $\mathscr{A}_{k}$-measurable function $g_{k}$ in $L^{p}$-norm as $n$ tends to infinity. For each $n, \mathscr{E}_{k}\left(f_{n}\right)$ is also valued in $K$, hence so is $g_{k}$ (almost everywhere). Now by (1), we have that $\left\|\mathscr{E}_{k} f_{n}-f_{k}\right\|_{p} \leqq \delta_{k}$ for all $n$, hence $\left\|g_{k}-f_{k}\right\|_{p}=$ $\lim _{n \rightarrow \infty}\left\|\mathscr{E}_{k} f_{n}-f_{k}\right\|_{p} \leqq \delta_{k}$. Thus (a) and (b) are established. To see that $\left(g_{n}\right)$ is a martingale, again fix $k$; then

$$
\begin{aligned}
\mathscr{E}_{k} g_{k+1} & =\mathscr{E}_{k} \lim _{n \rightarrow \infty} \mathscr{E}_{k+1} f_{n}=\lim _{n \rightarrow \infty} \mathscr{E}_{k} \mathscr{E}_{k+1} f_{n} \\
& =\lim _{n \rightarrow \infty} \mathscr{E}_{k} f_{n}=g_{k} .
\end{aligned}
$$

The final assertion is an immediate consequence of the following result.

THEOREM 1.3. Let $\left(f_{n}\right)$ be an $X$-valued quasi-martingale, $f$ a member of $L^{1}(X)$, and suppose $f_{n} \rightarrow f$ in $L^{1}(X)$. Then $f_{n} \rightarrow f$ a.e.

Indeed, the difference of two quasi-martingales (with respect to $\left.\left(\mathscr{A}_{j}\right)\right)$ is also a quasi-martingale. Thus $\left(f_{n}-g_{n}\right)$ of Theorem 1.1 is a quasi-martingale, and $f_{n}-g_{n} \rightarrow 0$ in $L^{1}$ norm by (b). Hence $f_{n}-g_{n} \rightarrow 0$ a.e. Of course Theorem 1.3 also follows from Theorem 1.1 and the validity of the corresponding martingale result. We prefer, however, to give a direct proof of Theorem 1.3 (thus including the "simpler" version of the martingale convergence theorem).

We first recall the notion of a stopping time (with respect to $\left.\left(. \mathscr{L}_{j}\right)\right)$. A function $\tau: \Omega \rightarrow\{0,1,2, \cdots\} \cup\{\infty\}$ is called a stopping time 
if $[\tau=j] \in \cdot /_{j}$ for all finite $j=0,1,2, \cdots$. Let $\tau$ be a stopping time, let $\left(f_{n}\right)$ satisfy the hypotheses of Theorem 1.3 with $\left(f_{n}\right)$ corresponding to $\left(\varepsilon_{n}\right)$, and let $f_{\infty}=f$. The function $f_{\approx}$ defined by $\left(f_{\tau}\right)(\omega)=$ $f_{\tau(\omega)}(\omega)$ shall be termed the quasi-martingale $\left(f_{n}\right)$ stopped at $\tau$. Our next result shows that the $L^{1}$-norm of a stopped quasi-martingale is dominated by that of the martingale plus the sum of the perturbing sequence.

LEMMA 1.4. $\left\|f_{i}\right\|_{1} \leqq\|f\|_{1}+\sum_{j=0}^{\infty} \varepsilon_{j}$.

Proof. Let $E_{j}=[\tau=j]$ for all $j$ and let $E=\Omega \sim E_{\infty}$; thus $E=\bigcup_{j=0}^{\infty} E_{j}$. Let $d_{0}=f_{0}$ and $d_{n}=f_{n}-f_{n-1}$ for all $n \geqq 1$. Our hypotheses assert that $\left\|\mathscr{E}_{j}\left(d_{j+1}\right)\right\| \leqq \varepsilon_{j}$ for all $j$. For each finite $j$ we have that

$$
\begin{aligned}
& \int_{E_{i}}\left\|f_{\tau}(\omega)\right\| d P(\omega)=\int_{E_{j}}\left\|\left(d_{0}+\cdots+d_{j}\right)(\omega)\right\| d P(\omega) \\
&=\int_{E_{j}} \| \mathscr{E}_{j}\left(d_{0}+\cdots+d_{j}+\cdots+d_{n}+\cdots\right) \\
& \quad-\mathscr{E}_{j}\left(d_{j+1}+\cdots+d_{j}+\cdots\right) \| d P(\omega) \\
&=\int_{E_{j}}\left\|\mathscr{O}_{j} f-\sum_{k=j+1}^{\infty} \mathscr{O}_{j}\left(d_{k}\right)\right\| d P(\omega) \\
& \leqq \int_{E_{j}}\left[\|f(\omega)\| d P(\omega)+\int_{E_{j} j} \sum_{k=j+1}^{\infty}\left\|\mathscr{E}_{j}\left(d_{k}\right)(\omega)\right\| d P(\omega)\right] .
\end{aligned}
$$

Thus

$$
\int_{E}\left\|f_{-}(\omega)\right\| d P(\omega) \leqq \int_{I}\|f(\omega)\| d P(\omega)+\sum_{\langle(j, k) ; j<k\}} \int_{l_{j} j}\left\|\mathscr{G}_{j}\left(d_{k}\right)(\omega)\right\| d P(\omega) .
$$

The second term equals $\sum_{k=1}^{\infty} \sum_{j<k} \int_{F_{j}}\left\|\mathscr{E}_{j}\left(d_{k}\right)(\omega)\right\| d P(\omega)$.

Now for each $j<k$,

$$
\begin{aligned}
& \int_{E_{j} j}\left\|\mathscr{G}_{j}\left(d_{k}\right)(\omega)\right\| d P(\omega)=\left\|\mathscr{\sigma}_{j}\left(\chi_{E_{j}} d_{l_{k}}\right)\right\|_{1} \leqq\left\|\mathscr{G}_{k-1}\left(\chi_{E_{j}} d_{k}\right)\right\|_{1} \\
& =\int_{E_{j}}\left\|\mathscr{f}_{k-1}\left(d_{k}\right)(\omega)\right\| d P(\omega) .
\end{aligned}
$$

$\left(\chi_{F}\right.$ denotes the indicator function of the set $F ; \chi_{F}(\omega)=1$ if $\omega \in F$, $\chi_{F}(\omega)=0$ otherwise.) Applying (3) we obtain

$$
\begin{gathered}
\sum_{k=1}^{\infty} \sum_{j<k} \int_{E_{j}}\left\|\mathscr{E}_{j}\left(d_{k}\right)(\omega)\right\| d P(\omega) \leqq \sum_{k=1}^{\infty} \sum_{j<k} \int_{E j}\left\|\mathcal{f}_{k-1} d_{k}(\omega)\right\| d P(\omega) \\
\leqq \sum_{k=1}^{\infty}\left\|\mathscr{O}_{k-1} d_{k}\right\|_{1}=\sum_{j=0}^{\infty} \varepsilon_{j} .
\end{gathered}
$$

Since $f_{:}=f$ on $E_{\infty}$, the above estimate and (2) complete the proof. 
We next pass to the standard martingale maximal inequality of J. Doob phrased in terms of quasi-martingales.

LEMma 1.5. Let $f^{*}=\sup _{j}\left\|f_{j}(\omega)\right\|$. Then for all $\lambda>0, \lambda P\left[f^{*}>\right.$ $\lambda] \leqq\|f\|_{1}+\sum_{j=0}^{\infty} \varepsilon_{j}$.

Proof. Let $\lambda>0$ and define $\tau$ by $\tau(\omega)=$ least $j$ such that $\left\|f_{j}(\omega)\right\|>\lambda$, if such exists; else $\tau(\omega)=\infty$. Then $\tau$ is indeed a stopping time. If $f^{*}(\omega)>\lambda$, then $\tau(\omega)<\infty$ and $\left\|f_{\tau}(\omega)\right\|>\lambda$ also; hence $\lambda P\left[f^{*}>\lambda\right] \leqq \int\left\|f_{\tau}(\omega)\right\| d P(\omega) \leqq\|f\|_{1}+\sum_{j=0}^{\infty} \varepsilon_{j}$ by Lemma 4 .

Proof of Theorem 1.3. Follow the same notation as in Lemma 1.5. Let $\lambda>0$. It suffices to show that $D=\left[\left\|f-f_{m}\right\|>\lambda\right.$ for infinitely many $m]$ has measure zero. Now fix $n$ and suppose $m>n$. Then $\left[\left\|f-f_{m}\right\|>\lambda\right] \subset\left[\sup _{l>m}\left\|d_{m+1}+\cdots+d_{l}\right\|>\lambda\right] \subset\left[\sup _{l>n} \| d_{n+1}+\cdots\right.$ $+d_{l} \|>\lambda / 2$ ] (up to sets of measure zero). Thus we have

$$
D \subset\left[\sup _{l>n}\left\|d_{n+1}+\cdots+d_{l}\right\|>\lambda / 2\right] .
$$

Now set $g_{l}=d_{n+1}+\cdots+d_{l}$ for $l \geqq n+1$ and $g_{l}=0$ for $0 \leqq l \leqq n$. Then $\left(g_{l}\right)$ is a quasi-martingale corresponding to the sequence $\left(\delta_{j}\right)$ with $\delta_{j}=\varepsilon_{j}$ if $j \geqq n$ and $\delta_{j}=0$ if $j<n$, and of course $\left(g_{l}\right)$ tends to $f-f_{n}$ in $L^{1}(X)$ as $l$ tends to infinity. Hence by (4) and Lemma $1.5, \lambda / 2 P(D) \leqq\left\|f-f_{n}\right\|_{1}+\sum_{j=n}^{\infty} \varepsilon_{j}$.

This shows $P(D)=0$ since $\lim _{n \rightarrow \infty}\left(\left\|f-f_{n}\right\|_{1}+\sum_{j=n}^{\infty} \varepsilon_{j}\right)=0$.

REMARK. If $X$ has the RNP and $\left(f_{n}\right)$ is an $L^{1}$-bounded $X$-valued quasi-martingale, then $\left(f_{n}\right)$ converges a.e. $\left(\left(f_{n}\right)\right.$ is $L^{1}$-bounded means $\sup _{n}\left\|f_{n}\right\|_{1}<\infty$.) Indeed by Theorem 1.3, there is a martingale $\left(g_{n}\right)$ and a sequence $\left(u_{n}\right)$ with $u_{n} \rightarrow 0$ in $L^{1}$ and a.e. so that $f_{n}=g_{n}+u_{n}$ for all $n$. Then $\left(g_{n}\right)$ is also $L^{1}$-bounded, hence $\left(g_{n}\right)$ converges a.e. (This result is also a special case of a known result of Bellow concerning uniform amarts [1].)

The final result of this section may be interpreted as follows: Suppose $K$ is a closed bounded convex subset of a Banach space so that $K$ has the RNP; suppose $\left(f_{n}\right)$ is a uniformly bounded quasimartingale with $f_{0} \in K$. Stop the martingale the first time it exits $K$. I.e. let $\tau$ equal the least $n$ so that $f_{n} \notin K$. Then the stopped martingale $\left(f_{\tau}\right)$ converges a.e. Quasi-martingales enter in a natural way in the proof (unless of course $X$ itself has the RNP).

Proposition 1.6. Let $K$ be a closed bounded convex subset of 
$X$ so that $K$ has the RNP. Let $\left(f_{n}\right)$ be a uniformly bounded $X$ valued quasi-martingale so that for all $\omega$ and $n$, if $f_{n}(\omega) \notin K$ then $f_{n+1}(\omega)=f_{n}(\omega)$. Then $\left(f_{n}\right)$ converges a.e.

Proof. Let us suppose $\left(f_{n}\right)$ is a quasi-martingale with respect to $\left(\mathscr{A}_{n}\right)$ corresponding to $\left(\varepsilon_{n}\right)$; as before, $\mathscr{E}_{n}$ denotes conditional expectation with respect to $\mathscr{A}_{n}$ and $\mathscr{A}_{0}$ is the trivial algebra. Let $G_{n}=\left[f_{n} \in K\right]$. Then our assumptions imply that $G_{n} \supset G_{n+1}$ for all $n$; hence $\sum_{n=0}^{\infty} P\left(G_{n} \sim G_{n+1}\right)<\infty$. We shall show that $\left(f_{n} \cdot \chi_{G_{n}}\right)$ is a quasi-martingale. Once this is done, we obtain that $\left(f_{n} \cdot \chi_{G_{n}}\right)$ converges a.e. by Corollary 1.2. But our assumptions yield immediately that $\left(f_{n} \cdot \chi_{B_{n}}\right)$ converges a.e., where $B_{n}=\sim G_{n}$ for all $n$. Hence $\left(f_{n}\right)$ converges a.e.

Let $M=\sup _{n}\left\|f_{n}\right\|_{\infty}$. Fix $n \geqq 0$. Then

$$
\begin{aligned}
\left\|\mathscr{E}_{n}\left(f_{n+1} \chi_{G_{n+1}}\right)-f_{n} \cdot \chi_{G_{n}}\right\|_{1} \\
\quad \leqq\left\|\mathscr{E}_{n}\left(f_{n+1} \chi_{G_{n}}\right)-f_{n} \cdot \chi_{G_{n}}\right\|_{1}+\left\|\mathscr{E}_{n} f_{n+1} \chi_{G_{n+1}}-\mathscr{E}_{n} f_{n+1} \chi_{G_{n}}\right\|_{1} \\
\quad=\left\|\left(\mathscr{E}_{n} f_{n+1}-f_{n}\right) \chi_{G_{n}}\right\|_{1}+\left\|\mathscr{E}_{n}\left[f_{n+1}\left(\chi_{G_{n+1}}-\chi_{G_{n}}\right)\right]\right\|_{1} \\
\quad \leqq\left\|\mathscr{E}_{n} f_{n+1}-f_{n}\right\|_{1}+\left\|f_{n+1}\left(\chi_{G_{n+1}}-\chi_{G_{n}}\right)\right\|_{1} \\
\quad \leqq \varepsilon_{n}+M P\left(G_{n} \sim G_{n+1}\right) .
\end{aligned}
$$

Hence $\sum_{n=0}^{\infty}\left\|\mathscr{E}_{n}\left(f_{n+1} \chi_{G_{n+1}}\right)-f_{n} \cdot \chi_{G_{n}}\right\|_{1} \leqq \sum_{n=0}^{\infty} \varepsilon_{n}+M \sum_{n=0}^{\infty} P\left(G_{n} \sim G_{n+1}\right)<$ $\infty$, thus $\left(f_{n} \cdot \chi_{G_{n}}\right)$ is a quasi-martingale, completing the proof.

REMARK. We are indebted to A. Bellow for pointing out that our argument yields that Proposition 1.6 holds if we replace the word "uniformly" by the word " $L$-bounded" in its statement. Indeed, the assumptions then imply by standard arguments that there is an integrable function $g$ with $\left\|f_{n}(\omega)\right\| \leqq g$ for all $n$ and $\omega$. Hence we obtain

$$
\begin{array}{cc}
\left\|\mathscr{E}_{n} f_{n+1} \chi_{G_{n+1}}-f_{n} \cdot \chi_{G_{n}}\right\|_{1} \leqq\left\|\mathscr{E}_{n} f_{n+1}-f_{n}\right\|_{1}+\left\|f_{n+1}\left(\chi_{G_{n+1}}-\chi_{G_{n}}\right)\right\|_{1} \\
\leqq \varepsilon_{n}+\left\|g\left(\chi_{G_{n+1}}-\chi_{G_{n}}\right)\right\|_{1}, & \text { as above } \\
\sum_{n=0}^{\infty}\left\|\mathscr{E}_{n}\left(f_{n+1} \chi_{G_{n+1}}\right)-f_{n} \chi_{G_{n}}\right\|_{1} \leqq \sum_{n=0}^{\infty} \varepsilon_{n}+\|g\|_{1}<\infty . & \text { so again }
\end{array}
$$

2. Denting points and strongly exposed points in RNP sets. We begin by establishing a standard result concerning the equivalence of nondentability of closed bounded convex sets and the existence of " $\delta$-bushes", or martingales with differences bounded everywhere away from 0 by $\delta$. The purpose is to develop technique and machinery for later results, and to show the "naturalness" of quasi-martingales in the setting of nondentability. 
Let $X$ be a fixed Banach space; for $x \in X$ and $r>0$, let $B_{x, r}=$ $\{y \in X:\|y-x\| \leqq r\}$. Let $K$ be a bounded subset of $X$. $K$ is said to be dentable if for every $\varepsilon>0$, there exists a $k \in K$ with $k \notin \overline{\operatorname{co}}\left(K \sim B_{k, \varepsilon}\right)$. It can be seen (see [6]) that $K$ is dentable if and only if $\overline{c o} K$ is dentable. ( $\overline{c o} A$ denotes the closed convex hull of the set $A$.)

Proposition 2.1. Let $K$ be a closed bounded convex set, $\delta>0$ and suppose $k \in \overline{\mathrm{co}}\left(K \sim B_{k, \delta}\right)$ for all $k \in K$. Let $\left(\varepsilon_{j}\right)_{j=0}^{\infty}$ be a summable sequence of positive numbers. Then there exists an $\infty$-quasi-martingale $\left(f_{n}\right)$ corresponding to $\left(\varepsilon_{n}\right)$, valued in $K$, with $\left\|\left(f_{n+1}-f_{n}\right)(\omega)\right\| \geqq$ $\delta$ for all $\omega$ and $n=0,1,2, \cdots$.

We first note a simple consequence.

CoRollaRy 2.2. Let $K, \delta$ as above and let $\delta^{\prime}<\delta$. There exists a martingale $\left(g_{n}\right)$ valued in $K$ with $\left\|\left(g_{n+1}-g_{n}\right)(\omega)\right\| \geqq \delta^{\prime}$ for all $\omega$ and $n=0,1,2, \cdots$.

Proof. Let $\left(\varepsilon_{j}\right)$ be such that $\sum_{j=0}^{\infty} \varepsilon_{j} \leqq\left(\delta-\delta^{\prime}\right) / 2$. Let $\delta_{n}=\sum_{j=n}^{\infty} \varepsilon_{j}$ for all $n=0,1,2, \cdots$. Let $\left(g_{n}\right)$ be the martingale associated with $\left(f_{n}\right)$, as given in the statement of Theorem 1.1. It follows from (b) of Theorem 1.1 that for all $\omega$ and $n$,

$$
\begin{aligned}
\left\|\left(g_{n+1}-g_{n}\right)(\omega)\right\| & \geqq\left\|\left(f_{n+1}-f_{n}\right)(\omega)\right\|-\left\|f_{n+1}-g_{n+1}\right\|_{\infty}-\left\|f_{n}-g_{n}\right\|_{\infty} \\
& \geqq \delta-\delta_{n+1}-\delta_{n} \geqq \delta-2 \delta_{0} \geqq \delta^{\prime}
\end{aligned}
$$

REMARK. The proof of Proposition 2.1 will show that the martingale $\left(g_{n}\right)$ is such that $g_{n}$ is finite valued for all $n$ (and also that each value is taken on with positive probability). Call such a martingale a nice martingale. (Thus, $\left(g_{n}\right)$ corresponds precisely to a " $\delta$-bush" as described in [6].) Corollary 2.2 thus produces the known result that the following two mutually exclusive alternatives hold for a closed bounded convex set $K$ : either every nonempty subset of $K$ is dentable, or there exists a $\delta^{\prime}>0$ and a nice martingale $\left(g_{n}\right)$ valued in $K$ satisfying the conclusion of Corollary 2.2. Indeed, if $L=\left\{g_{n}(\omega): \omega \in \Omega, n=0,1,2, \cdots\right\}$, then $L$ is not dentable. For given $n$ and $\omega$, let $A=\left\{\omega^{\prime}: g_{n}\left(\omega^{\prime}\right)=g_{n}(\omega)\right\}$; then, by definition,

$$
g_{n}(\omega)=\mathscr{E}_{n} g_{n+1}(\omega)=\frac{1}{P(A)} \int_{A} g_{n+1} d P
$$

The last term is in the convex hull of $\left\{g_{n+1}\left(\omega^{\prime}\right): \omega^{\prime} \in A\right\}$; and of course $g_{n}(\omega)=g_{n}\left(\omega^{\prime}\right)$ with $\left\|g_{n}\left(\omega^{\prime}\right)-g_{n+1}\left(\omega^{\prime}\right)\right\| \geqq \delta^{\prime}$ for all $\omega^{\prime} \in A$. (We freely use the deeper fact that every nonempty subset of $K$ is dentable if and only if $K$ has the RNP.) 
Proof of Proposition 2.1. We shall take the half-open unit interval $[0,1)$ with Lebesgue measurable sets and Lebesgue measure, as our probability space. We shall construct $\left(f_{n}\right)$ corresponding to $\left(\mathscr{A}_{n}\right)$, where for each $n, \mathscr{A}_{n}$ is a finite algebra generated by halfopen intervals.

Let $\mathscr{A}_{0}$ equal the trivial algebra. Let $k_{0} \in K$ be arbitrary and let $f_{0}(\omega)=k_{0}$ for all $\omega$. Suppose $n \geqq 0$ and $\mathscr{A}_{n}, f_{n}$ have been defined. Choose $k$ and $0=c_{0}<c_{1}<\cdots<c_{k}=1$ (depending on $n$ ) so that - $\mathscr{A}_{n}$ is generated by $\left\{\left[c_{j-1}, c_{j}\right): 1 \leqq j \leqq k\right\}$. Fix $j$, set $A=\left[c_{j-1}, c_{j}\right)$ and let $k_{A}$ be the value of $f_{n}$ on $A$. Now by assumption we may choose elements $k_{1}, \cdots, k_{m}$ in $K$ and positive numbers $\lambda_{1}, \cdots, \lambda_{m}$ with $\left\|k_{i}-k_{A}\right\| \geqq \delta$ for all $i$ and $\left\|\sum_{i=1}^{m} \lambda_{i} k_{i}-k_{A}\right\|<\varepsilon_{n}$. Set $d_{0}=c_{j-1}$ and define $d_{1}, \cdots, d_{m}$ successively by $d_{i}-d_{i-1}=\lambda_{i}\left(c_{j}-c_{j-1}\right)$. It follows that $c_{j-1}=d_{0}<d_{1}<\cdots<d_{m}=c_{j}$. (Of course the quantities $k, m, k_{1} \cdots k_{m}$ and $d_{0}, d_{1} \cdots d_{m}$ all depend on $j$; we simply avoid the cumbersome indexing.) Now let $\mathscr{A}_{n+1}$ be the algebra generated by $\mathbf{U}_{j=1}^{k}\left\{\left[d_{i-1}, d_{i}\right): 1 \leqq i \leqq m\right\}$. Again fixing $j$, define $f_{n+1}$ on $\left[c_{j-1}, c_{j}\right)$ by $f_{n+1}=\sum_{i=1}^{m} k_{i} \chi_{\left[d_{i-1}, d_{i}\right)}$. Then for $\omega \in\left[c_{j-1}, c_{j}\right)$,

$$
\left(\mathscr{E}_{n} f_{n+1}\right)(\omega)=\frac{1}{c_{j}-c_{j-1}} \int_{c_{j-1}}^{c_{j}} f_{n+1} d x=\sum_{i=1}^{m} \lambda_{i} k_{i}
$$

and hence $\left\|\mathscr{E}_{n} f_{n+1}-f_{n}\right\|_{\infty} \leqq \varepsilon_{n}$ and $\left\|f_{n+1}-f_{n}(\omega)\right\| \geqq \delta$ for all $\omega$. This completes the construction of $\left(f_{n}\right)$ and $\left(\mathscr{L}_{n}\right)$ by induction, and hence the proof of 2.1 .

We next treat the case of denting points of RNP sets $K$. We shall later consider the case of strongly exposed points; this case yields the denting point result. However the basic technique seems more clearly revealed in the simpler setting of denting points. We recall that if $K$ is a nonempty bounded subset of a Banach space $X$, then $x \in K$ is called a denting point of $K$ if $x \notin \overline{\mathrm{co}}\left(K \sim B_{x, \varepsilon}\right)$ for all $\varepsilon>0$. A subset $S$ of $K$ is called a slice of $K$ if there is an $f \in X^{*}$ with $\|f\|=1$ and a $\delta>0$ so that $S=\{k \in K: f(k) \geqq \sup f(K)-\delta\}$. In this case, we write $S=S(f, \delta)$.

We wish to present a martingale proof of the following known result (c.f. [2] and [9]):

THEOREM 2.2. Let $K$ be a closed bounded convex set in the Banach space $X$ and suppose $K$ has the RNP. Then $K$ equals the closed convex hull of its set of denting points.

The main lemma in the proof is as follows:

Lemma 2.3. Let $K$ be as in Theorem 2.2, let $S$ be a slice of $K$, 
and let $\varepsilon>0$. There exists a slice $\widetilde{S}$ of $K$ with diameter $\widetilde{S} \leqq 2 \varepsilon$ and $\widetilde{S} \subset S$.

Let us first quickly sketch the (well-known) proof that $2.3 \Rightarrow 2.2$. Suppose the claim is false; let $W$ equal the closed convex hull of the set of denting points of $K$. ( $W$ could be empty, of course.) Since $W \neq K$, it follows from the Hahn-Banach theorem that there is a slice $S$ of $K$ with $W \cap S=\varnothing$. By 2.3, we may 'choose slices $S_{1} \subset S, S_{2} \subset S_{1}, \cdots, S_{n+1} \subset S_{n}$ of $K$ with diam $S_{n}<1 / n$ for all $n$ (where $\operatorname{diam} L=$ diameter of $L)$. It follows that there is an $x \in \bigcap_{i=1}^{\infty} S_{j} ; x$ is a denting point of $K$. But since $x \in S, x \notin W$, a contradiction.

The next lemma easily yields 2.3, and is directly accessible via martingale techniques.

LEMMA 2.4. Let $K$ be as in 2.2, $f \in X^{*}$ with $\|f\|=1,0<\eta<\alpha$ and $\varepsilon>0$. Assume $K \subset B_{0,1}$ and $\varepsilon<\alpha-\eta$. There exists a $k_{0} \in$ $S(f, \eta)$ with $k_{0} \notin W=\overline{c o}\left(S(f, \alpha) \sim B_{k_{0}, \varepsilon}\right)$.

Proof that $2.4 \Longrightarrow 2.3$. Of course we can assume without loss of generality that $K \subset B_{0,1}$. Choose $f$ and $\alpha>0$ with $S=S(f, \alpha)$ and set $M=\sup f(K)$. Choose $\eta<\alpha$ and assume $\varepsilon<\alpha-\eta$. Choose $k_{0}$ as in 2.4. Now let $g$ be a norm-one functional separating $k_{0}$ from $W$; i.e., $\sup g(W)<g\left(k_{0}\right)$. Choose $\tau$ with $\sup g(W)<\tau<g\left(k_{0}\right)$ and set $\widetilde{S}=\{k \in K: g(k) \geqq \tau\}$. It's obvious that $\widetilde{S}$ is a slice of $K$. It suffices to show that $\widetilde{S} \subset S$. Indeed, once this is done, since $k_{0} \in \widetilde{S}$, $k \in \widetilde{S}$ implies $\left\|k-k_{0}\right\|<\varepsilon$ since $k \notin W$, so $\widetilde{S}$ has diameter at most $2 \varepsilon$.

So suppose $k \in \widetilde{S}$ yet $k \notin S$. Thus $f(k)<M-\alpha$. Now the straight line segment joining $k$ to $k_{0}$ lies in $\widetilde{S}$; it follows that there exists a $\bar{k}$ on this segment with $f(\bar{k})=M-\alpha$. But then $\varepsilon<\alpha-$ $\eta \leqq f\left(k_{0}\right)-f(\bar{k}) \leqq\left\|k_{0}-\bar{k}\right\|$. Thus $\bar{k} \in W \cap \widetilde{S}$, but $W \cap \widetilde{S}=\varnothing$, a contradiction.

We pass finally to our first "serious" use of martingales for the geometry of Banach spaces. We shall take all quasi-martingales with respect to some fixed probability space $(\Omega, \mathscr{S}, P)$; of course the "standard" unit-interval space will serve.

Proof of Lemma 2.4. Assume the conclusion is false. Let $k_{0} \in$ $K$ be such that

$$
f\left(k_{0}\right)>M-\eta \text { where } M=\sup f(K) \text {. }
$$

It follows that we may obtain $k_{0}$ as almost equal to a convex combination of elements $k_{1}, \cdots, k_{n}$ of $S(f, \alpha)$, each of which is at 
least $\varepsilon$ away from $k_{0}$. Now for each $i$, if $k_{i} \notin S(f, \eta)$, we stop this process. Otherwise, we again express $k_{i}$ as almost equal to a convex combination of elements of $S(f, \alpha)$, all at least $\varepsilon$ away from $k_{i}$. Continuing in this way, we obtain an $\infty$-quasi-martingale starting at $k_{0}$, a point strictly above the hyperplane $\{x: f(x)=M-\eta\}$, yet which converges almost everywhere to a function $h$ which is everywhere below this hyperplane. But the expected value of $h$ must be almost equal to $k_{0}$ and hence lie above the hyperplane, a contradiction.

The precise argument goes as follows: Let $\left(\varepsilon_{j}\right)_{j=0}^{\infty}$ be a summable sequence of positive numbers so that

$$
\delta_{0}=\sum_{j=0}^{\infty} \varepsilon_{j}<f\left(k_{0}\right)-M+\eta .
$$

Thus

$$
f\left(k_{0}\right)-\delta_{0}>M-\eta
$$

Now by the same technique as in the proof of Proposition 2.1, we may choose an $\infty$-quasi-martingale $\left(h_{n}\right)$ corresponding to $\left(\varepsilon_{n}\right)$ with the following properties for all $\omega$ and $n=0,1, \cdots$ :

$$
h_{0} \equiv k_{0}
$$

((9) is not crucial to our argument.)

Now since $K$ has the RNP, $h_{n}$ converges almost everywhere to a function $h$, by Corollary 1.2. It then follows by (8) that $f(h(\omega))<$ $M-\eta$ a.e. $\omega$, and hence

$$
\int f(h(\omega)) d P<M-\eta
$$

(since $\int h_{n}(\omega) d P \rightarrow \int h(\omega) d P$ by the bounded convergence theorem). By the remark following the statement of Theorem 1.1, we have that $\left\|\int h_{n} d P-k_{0}\right\| \leqq \delta_{n}+\delta_{0}$ for all $n$ and hence

$$
\left\|\int h d P-k_{0}\right\| \leqq \delta_{0}
$$

From (11) we obtain that $\int f(h) d P \geqq f\left(k_{0}\right)-\delta_{0}>M-\eta$ by (6), contradicting (10).

REMARK. It seems worth pointing out that the proof of Lemma 
2.4 actually gives an effective procedure for finding the desired $k_{0}$. The argument is also accessible without passing to the martingale convergence theorem; the $L^{1}$-norms of the differences of the sequence $\left(h_{n}\right)$ will be strictly bounded away from 0 , and hence $\left(h_{n}\right)$ will not converge in $L^{1}$-norm, contradicting (c) of Corollary 1.2. Let us see why this is so. Let $\tau=f\left(k_{0}\right)-M+\eta ; \tau>\delta_{0}$ by (6). Fix $n$ and let $B_{n}=\left[f\left(h_{n}\right) \geqq M-\eta\right]$. By the inequality preceding (11) we obtain that

$$
\int f\left(h_{n}(\omega)\right) d P \geqq f\left(k_{0}\right)-\delta_{n}-\delta_{0}=M-\eta+\tau-\delta_{n}-\delta_{0} .
$$

But

$$
\begin{aligned}
\int f\left(h_{n}(\omega)\right) d P= & \int_{B_{n}} f\left(h_{n}(\omega)\right) d P+\int_{\sim B_{n}} f\left(h_{n}(\omega) d P \leqq M P\left(B_{n}\right)\right. \\
& +(M-\eta)\left(1-P\left(B_{n}\right)\right) .
\end{aligned}
$$

Combining (12) and (13), we obtain that $\left(\tau-\delta_{n}-\delta_{0}\right) / \eta \leqq P\left(B_{n}\right)$; since $\delta_{n} \rightarrow 0$, we have that $P\left(B_{n}\right) \geqq\left(\tau-\delta_{0}\right) / 2 \eta$ for all $n$ large enough. But then for such $n$,

$$
\begin{aligned}
\left\|h_{n+1}-h_{n}\right\|_{1} & \geqq \int_{B_{n}}\left\|h_{n+1}-h_{n}\right\| d P \\
& \geqq \varepsilon P\left(B_{n}\right) \text { by }(8) \\
& \geqq \frac{\varepsilon\left(\tau-\delta_{0}\right)}{2 \eta}, \text { a contradiction. }
\end{aligned}
$$

We next proceed to the most delicate consideration treated here, that of strongly exposed points. Fix $K$ a closed bounded convex subset of a Banach space $X$. A point $k \in K$ is said to be a strongly exposed point of $K$ if there exists an $f \in X^{*}$ with $\|f\|=1$ so that

$$
\begin{aligned}
& f(k)=\sup f(K) \text { and for any sequence }\left(k_{n}\right) \in K, \text { if } \\
& f\left(k_{n}\right) \rightarrow f(k) \text { then } k_{n} \rightarrow k \text { in norm. }
\end{aligned}
$$

It is evident that if $k$ is a strongly exposed point of $K$, then $k$ is a denting point of $K$. Indeed, (14) is equivalent to the assertion that for all $\varepsilon>0$, there is an $\alpha>0$ so that $k \in S(f, \alpha)$ and $\operatorname{diam} S(f, \alpha)<\varepsilon$. Thus the following theorem of J. Bourgain [2] implies Theorem 2.2.

THEOREM 2.5. Let $K$ be a closed bounded convex set with the RNP. Then $K$ equals the closed convex hull of its set of strongly exposed points. 
Following the terminology of Bourgain, we say that an $f \in X^{*}$ with $\|f\|=1$ is a strongly exposing linear functional for $K$ if there is a $k \in K$ satisfying (14). We denote the set of all such $f \in X^{*}$ with $\|f\|=1$ by SELF. We proceed initially just as in [2]. (Assume $\left.K \subset B_{0,1}\right)$.

THEOREM 2.6. SELF is a norm-dense $G_{i}$-subset of the unit sphere of $X^{*}$.

(The unit sphere of $X^{*}$ is by definition the set of all $f \in X^{*}$ with $\|f\|=1$.)

Let us quickly sketch the proof that $2.6 \Rightarrow 2.5$. Suppose 2.5 were false and let $W$ equal the closed convex hull of its set of its strongly exposed points. Let $g \in X^{*}$ with $\|g\|=1$ so that $g$ separates $W$ from some point in $K$. Thus $\sup g(W)<M=\sup g(K)$. Now let $\varepsilon>0$, to be decided later, and choose $f \in \mathrm{SELF}$ with $\|f-g\|<$ $\varepsilon$; also let $k \in K$ satisfy (14). Then $g(k)>f(k)-\varepsilon$ and $f(k)>M-\varepsilon$. Hence $g(k)>M-2 \varepsilon$; so as long as $M-2 \varepsilon>\sup g(W), k \notin W$ and we have a contradiction.

For each $\varepsilon>0$, let

$$
\begin{aligned}
A_{\varepsilon}=\left\{f \in X^{*}:\|f\|=1 \text { and there is an } \alpha>0\right. \text { with } \\
\\
\operatorname{diam} S(f, \alpha)<\varepsilon\} .
\end{aligned}
$$

We now arrive at the crucial geometrical result of Bourgain.

Lemma 2.7. $A_{\beta}$ is a dense subset of the unit sphere of $X^{*}$ for every $\beta>0$.

It is easily seen that $2.7 \Rightarrow 2.6$. Indeed, for each $\beta>0, A_{\beta}$ is an open subset of the unit sphere of $X^{*}$ and SELF $=\bigcap_{n=1}^{\infty} A_{1 / n}$. Thus 2.6 follows immediately from 2.7 and the Baire category theorem.

At this point we part company with Bourgain's treatment and formulate instead a lemma which yields 2.7 and is amenable to martingale techniques.

LEMma 2.8. Let $f \in X^{*}$ with $\|f\|=1$ and $M=\sup f(K)$. Let $\varepsilon>0$ and let $0<\eta<\alpha$. There exists a $k_{0} \in S(f, \eta)$ so that

$$
k_{0} \notin W=\overline{\mathrm{co}}\left(\left(K \sim B_{k_{0}, \varepsilon}\right) \cup\left(B_{k_{0}, 1} \cap\{x \in X: f(x)<M-\alpha\}\right)\right) \text {. }
$$

We first present the proof that $2.8 \Rightarrow 2.7$; this is rather more involved than the argument that $2.4 \Rightarrow 2.3$.

Let $f \in X^{*}$ with $\|f\|=1$ and let $\delta$ and $\beta>0$. We shall show 
that there is a $g \in A_{\beta}$ with $\|g-f\|<\delta$. We may assume $K$ has more than one point, or else this is trivial. We may also assume without loss of generality, that $f$ assumes at least two values on $K$; indeed the Hahn-Banach theorem yields that the set of all such norm-one functionals is dense in the unit sphere of $X^{*}$.

Now let $\varepsilon$ be such that $2 \varepsilon<\beta, \alpha$ be such that $2 \alpha<\delta$, and also suppose $\eta, \varepsilon, \alpha$ satisfy

$$
\eta+\varepsilon+6 \alpha<\sup f(K)-\inf f(K) .
$$

Now choose $k_{0}$ as in the statement of 2.8 , then choose $g$ a normone functional so that $\sup g(W)<g\left(k_{0}\right)$. Evidently there is a $\gamma>0$ so that $k_{0} \in S(g, \gamma)$ and $W \cap S(g, \gamma)=\varnothing$. It follows that

$$
\left\|k-k_{0}\right\|<\varepsilon \text { for all } k \in S(g, \gamma),
$$

hence $g \in A_{\beta}$. We shall prove that $\|g-f\|<\delta$. Now since $g$ separates $k_{0}$ from $B_{k_{0}, 1} \cap\{x \in S: f(x)<M-\alpha\}, g$ separates 0 from

$$
\begin{aligned}
B_{k_{0}, 1} & \cap\{x \in X: f(x)<M-\alpha\}-k_{0} \\
& =B_{0,1} \cap\left\{x \in X: f(x)<M-\alpha-f\left(k_{0}\right)\right\} \supset B_{0,1} \cap\{x \in X: f(x)<-\alpha\} .
\end{aligned}
$$

Thus, suppose $x \in X,\|x\| \leqq 1$; then

$$
0=g(0)>g(x) \text { if } f(x)<-\alpha .
$$

This implies that

$$
f(x) \geqq-\alpha \text { if } g(x)=0,
$$

which in turn yields that

$$
|f(x)| \leqq \alpha \text { if }\|x\| \leqq 1 \text { and } g(x)=0 .
$$

Since $f$ and $g$ have norm-one, (17) and a lemma of Bishop and Phelps (see Lemma 2, page 188 of [6]) yield that

$$
\|f-g\| \leqq 2 \alpha \text {, or }\|f+g\| \leqq 2 \alpha .
$$

We finally give the technical details showing that by (15), the latter inequality is false.

Since $\sup g(K)=\sup g(S(g, \gamma)),(16)$ yields

$$
g\left(k_{0}\right) \geqq \sup g(K)-\varepsilon .
$$

Now suppose $\|f+g\| \leqq 2 \alpha$. This implies $|[\sup -g(K)]-M| \leqq$ $2 \alpha$ and $\left|-g\left(k_{0}\right)-f\left(k_{0}\right)\right| \leqq 2 \alpha$, hence

$$
\left|\inf g(K)-g\left(k_{0}\right)\right| \leqq 4 \alpha+M-f\left(k_{0}\right) \leqq \eta+4 \alpha .
$$

Combining (18) and (19), we obtain 


$$
\sup g(K)-\varepsilon \leqq g\left(k_{0}\right) \leqq \inf g(K)+\eta+4 \alpha,
$$

that is,

$$
\sup g(K)-\inf g(K) \leqq \eta+\varepsilon+4 \alpha .
$$

Then since $\|f+g\| \leqq 2 \alpha$, we obtain $\sup f(K)-\inf f(K) \leqq \eta+$ $\varepsilon+6 \alpha$, contradicting (15).

Proof of Lemma 2.8. We proceed in much the same way as in the proof of 2.4. Assume the conclusion is false. Let $k_{0} \in K$ be such that (5) holds. It follows that we may obtain $k_{0}$ as almost equal to a convex combination of elements $x_{1}, \cdots, x_{n}$ of $X$ so that for all $i$, either $x_{i} \in K$ and $\left\|x_{i}-k_{0}\right\| \geqq \varepsilon$ or $\left\|x_{i}-k_{0}\right\| \leqq 1$ and $f\left(x_{i}\right)<M-\alpha$. We note that

$$
\text { if } f(x)<M-\alpha \text {, then }\left\|x-k_{0}\right\|>\alpha-\eta \text {. }
$$

Indeed, $\alpha-\eta<f\left(k_{0}\right)-f(x) \leqq\|f\|\left\|k_{0}-x\right\|=\left\|k_{0}-x\right\|$. Hence for all $i,\left\|x_{i}-k_{0}\right\| \geqq \min \{\varepsilon, \alpha-\eta\}$. If $x_{i} \notin S(f, \eta)$, we stop this process; otherwise we "replace" $k_{0}$ with $x_{i}$ and repeat the process.

Precisely, let $\left(\varepsilon_{j}\right)$ be a summable sequence of positive numbers satisfying (6), where $\delta_{0}=\sum_{j=0}^{\infty} \varepsilon_{j}$. By the technique of the proof of Proposition 2.1, we may choose an $\infty$-quasi-martingale $\left(h_{n}\right)$ corresponding to $\left(\varepsilon_{n}\right)$ with the following properties for all $n=0,1,2, \cdots$ :

$$
h_{0} \equiv k_{0} \text {; }
$$

$$
\left\{\begin{array}{l}
\text { if } f\left(h_{n}(\omega)\right) \geqq M-\eta, \text { then } h_{n}(\omega) \in K \text { and } \\
\left\|h_{n+1}(\omega)-h_{n}(\omega)\right\| \geqq \varepsilon \text { or } \\
f\left(h_{n+1}(\omega)\right)<M-\alpha \text { and }\left\|h_{n}(\omega)-h_{n+1}(\omega)\right\| \leqq 1 ;
\end{array}\right.
$$$$
\text { if } f\left(h_{n}(\omega)\right)<M-\eta \text {, then } h_{n+1}(\omega)=h_{n}(\omega) \text {. }
$$

It follows from (22) and (20) that

$$
\text { if } f\left(h_{n}(\omega)\right) \geqq M-\eta \text {, then }\left\|h_{n+1}(\omega)-h_{n}(\omega)\right\| \geqq \min \{\varepsilon, \alpha-\eta\}>0 \text {. }
$$

Now the quasi-martingale $\left(h_{n}\right)$ is valued in $K+B_{0,1}$ and is hence uniformly bounded. Moreover $\left(h_{n}\right)$ "stops" when it leaves $K$, by (22) and (23); hence since $K$ has the RNP, $\left(h_{n}\right)$ converges almost everywhere to a function $h$, by Proposition 1.6. The rest of the argument is exactly the same as in the proof of Lemma 2.4. That is, $f(h(\omega))<M-\eta$ a.e., and hence (10) and (11) hold, yielding the contradiction given at the end of the proof of 2.4 .

3. E-strong extreme points and dyadic martingales. In this 
section we show how " $\delta$-trees" are intimately connected to a new quantitative notion of extreme point. As always, $X$ denotes a fixed Banach space.

Definition. Let $K$ be a bounded subset of $X$ and $\varepsilon>0$. A point $x \in K$ is called an $\varepsilon$-strong extreme point of $K$ if there is a $\delta>0$ so that if $k_{1}, k_{2}$ belong to $K$ and there is a point $u$ on the line segment joining $k_{1}$ and $k_{2}$ with $\|u-x\|<\delta$, then $\left\|u-k_{1}\right\|<\varepsilon$ or $\left\|u-k_{2}\right\|<\varepsilon$. The set of all $\varepsilon$-strong extreme points of $K$ is denoted by $E_{\varepsilon}(K)$.

\section{REMARKS.}

1. Let $K$ be convex. Then $x \in E_{\varepsilon}(K)$ if and only if there is a $\delta>0$ so that if $k_{1}, k_{2}$ belong to $K$ and $\left\|\left(\left(k_{1}+k_{2}\right) / 2\right)-x\right\|<\delta$, then $\left\|k_{1}-k_{2}\right\|<2 \varepsilon$.

2. Say that $x \in K$ is a strong extreme point if $x$ is an $\varepsilon$-strong extreme point for every $\varepsilon>0$. Evidently a strong extreme point is an extreme point; a denting point of a convex set is a strong extreme point of the set.

3. It is evident that $E_{\varepsilon}(K)$ is a relatively open subset of $K$. It follows that $E_{\varepsilon}(K) \neq \varnothing$ if $E_{\varepsilon}(\bar{K}) \neq \varnothing$.

It is useful to explicitly identify the complement of the set of $\varepsilon$-strong extreme points.

Definition. Let $\varepsilon>0, K$ a bounded subset of $X$, and let $G_{\varepsilon}(K)$ denote the set of all points $u \in X$ so that there exist $k_{1}, k_{2} \in K$ with $u$ on the line segment joining $k_{1}$ and $k_{2}$ with $\left\|u-k_{i}\right\| \geqq \varepsilon$ for $i=1$ and 2.

Proposition 3.1. Let $\varepsilon>0$. Then $E_{\varepsilon}(K)=K \sim \overline{G_{\varepsilon}(K)}$. Moreover if $K$ is convex, then

$$
G_{\varepsilon}(K)=\left\{\frac{k_{1}+k_{2}}{2}:\left\|k_{1}-k_{2}\right\| \geqq 2 \varepsilon \text { and } k_{i} \in K \text { for } i=1,2\right\} .
$$

The proof is an immediate consequence of the definitions.

We pass now to some generalizations of dyadic martingales. Let $(\Omega, \mathscr{S}, P)$ be a fixed probability space and $\left(\mathscr{X}_{j}\right)_{j=0}^{\infty}$ an increasing sequence of finite algebras of $\mathscr{S}$ with $\mathscr{A}_{0}$ the trivial algebra. Suppose that for all $j$ and $A \in \mathscr{A}_{j}$, if $P(A)=0$, then $A=\varnothing$. We shall say that $\left(\mathscr{A}_{j}\right)$ is a generalized dyadic decomposition of $\Omega$ if for all $n \mathscr{A}_{n}$ has $2^{n}$ atoms and no atom of $\mathscr{O}_{n}$ is an atom of $\mathscr{O}_{n+1}$. Let $0<\tau \leqq 1 / 2$. A generalized dyadic decomposition $\left(\mathscr{A}_{j}\right)$ of $\Omega$ is called a $\tau$-dyadic decomposition if for all $n$, all atoms $A$ of $\mathscr{A}_{n}$ and 
atoms $B$ of $\mathscr{A}_{n+1}$, if $B \varsubsetneqq A$ then $\tau \leqq P(B) / P(A)$ (so also $P(B) / P(A) \leqq$ $1-\tau)$. Of course a dyadic decomposition is simply a $1 / 2$-dyadic decomposition. A quasi-martingale $\left(f_{n}\right)$ shall be called dyadic (resp. $\tau$-dyadic, resp. generalized-dyadic) if it is a quasi-martingale with respect to a dyadic (resp. $\tau$-dyadic, resp. generalized-dyadic) decomposition.

REMARK. Suppose $\left(f_{n}\right)$ is an $X$-valued generalized dyadic martingale with $\left\|f_{n}(\omega)\right\| \leqq M$ and $\left\|f_{n+1}(\omega)-f_{n}(\omega)\right\| \geqq \delta$ for all $n$ and $\omega$. Then $\left(f_{n}\right)$ corresponds precisely to a "generalized $\delta$-tree", in Banach space terminology. Indeed, e.g., say $f_{0} \equiv k_{0}$; then $f_{1}$ is at most two valued; say with values $k_{1}$ and $k_{2}$ where $k_{0}=(1-\lambda) k_{1}+$ $\lambda k_{2}$ for some $0<\lambda<1$. Note that then $\left\|k_{0}-k_{1}\right\|=\lambda\left\|k_{2}-k_{1}\right\| \geqq \delta$, hence $\lambda \geqq \delta /\left\|\left(k_{2}-k_{1}\right)\right\| \geqq \delta / 2 M$. Thus $\left(f_{n}\right)$ is automatically a $\delta / 2 M$ dyadic martingale.

We are now prepared for our first result connecting $\varepsilon$-strong extreme points and dyadic martingales.

Proposition 3.2. Let $\delta>0, K$ a closed bounded convex set, and suppose that $K$ has no $\delta$-strong extreme points. Then for every $\delta^{\prime}<\delta$, there exists a dyadic martingale $\left(g_{n}\right)$ valued in $K$ so that $\left\|g_{n+1}(\omega)-g_{n}(\omega)\right\| \geqq \delta^{\prime}$ for all $n$ and $\omega$. That is, $K$ contains a $\delta^{\prime}-$ tree.

Proof. By Proposition 3.1, we have that

$$
\begin{aligned}
\overline{G_{\hat{o}}(K)} & =K, \text { where } G_{\dot{o}}(K) \\
& =\left\{\frac{k_{1}+k_{2}}{2}: k_{1}, k_{2} \in K \text { and }\left\|k_{1}-k_{2}\right\| \geqq 2 \delta\right\} .
\end{aligned}
$$

Let $\left(\mathscr{A}_{n}\right)$ be the standard dyadic decomposition of the half-open unit interval $[0,1)$; for each $n, \mathscr{A}_{n}$ is the algebra generated by $\left\{\left[(j-1) / 2^{n}, j / 2^{n}\right): 1 \leqq j \leqq 2^{n}\right\}$. Let $\delta^{\prime}<\delta^{\prime \prime}<\delta$. Let $\left(\varepsilon_{n}\right)$ be positive numbers so that

$$
\delta_{0}=\sum_{n=0}^{\infty} \varepsilon_{n} \leqq \frac{\delta^{\prime \prime}-\delta^{\prime}}{2} \text { and } \delta_{0} \leqq \delta-\delta^{\prime \prime} .
$$

We first construct an $\infty$-quasi-martingale $\left(f_{n}\right)$ corresponding to $\left(\varepsilon_{n}\right)$ (with respect to $\left(\mathscr{R}_{n}\right)$ ) valued in $K$ so that

$$
\left\|f_{n+1}(\omega)-f_{n}(\omega)\right\| \geqq \delta^{\prime \prime} \text { for all } n \text { and } \omega \text {. }
$$

Let $k_{0} \in K$ be arbitrary and set $f_{0} \equiv k_{0}$. Let $n \geqq 0$, suppose $f_{n}$ is defined, fix $1 \leqq j \leqq 2^{n}$ and let $k$ be the value of $f_{n}$ on $\left[(j-1) / 2^{n}\right.$, $\left.j / 2^{n}\right)$. By (25) we may choose $k_{1}, k_{2} \in K$ with $\left\|k-\left(\left(k_{1}+k_{2}\right) / 2\right)\right\| \leqq \varepsilon_{n}$ 
and $\left\|k_{1}-k_{2}\right\| \geqq 2 \delta$.

It follows that $\left\|k-k_{i}\right\| \geqq \delta-\varepsilon_{n} \geqq \delta^{\prime \prime}$ for $i=1,2$. Define $f_{n+1}$ on $\left[(j-1) / 2^{n}, j / 2^{n}\right)$ by

$$
f_{n+1}=k_{1} \text { on }\left[\frac{j-1}{2^{n}}, \frac{2 j-1}{2^{n+1}}\right) ; f_{n+1}=k_{2} \text { on }\left[\frac{2 j-1}{2^{n+1}}, \frac{j}{2^{n}}\right) \text {. }
$$

Now that $\left(f_{n}\right)$ has been constructed, let $\left(g_{n}\right)$ be the martingale (with respect to $\left(\mathscr{A}_{n}\right)$ ) given by Theorem 1.1. Since $\left(\mathscr{A}_{n}\right)$ is a dyadic decomposition, $\left(g_{n}\right)$ is a dyadic martingale. By the same argument as in the proof of Proposition 2.1, we obtain that for all $n$ and $\omega$,

$$
\left\|g_{n+1}(\omega)-g_{n}(\omega)\right\| \geqq \delta^{\prime \prime}-2 \delta_{0} \geqq \delta^{\prime} \text { by }(26),
$$

completing the proof.

Some of our motivation for introducing $\varepsilon$-strong extreme points for sets that are not necessarily convex came from the desire to obtain a kind of converse to the preceding result. In fact, we don't know the answer to the following question: suppose $K$ contains a $\delta^{\prime}$-tree for some $\delta^{\prime}>0$, i.e., there is a dyadic martingale $\left(g_{n}\right)$ valued in $K$ and satisfying the conclusion of Proposition 3.2. Is there a closed bounded convex nonempty subset $L$ of $K$ with $E_{\varepsilon}(L)=\varnothing$ for some $\varepsilon>0$ ?

Proposition 3.3. Let $K$ be a closed bounded convex subset of $X$. Then the following are equivalent:

(a) there is a nonempty subset $A$ of $K$ and $a \delta>0$ so that $A$ has no $\delta$-strong extreme points;

(b) there exists $a \delta^{\prime}>0$ and a generalized dyadic martingale $\left(g_{n}\right)$ valued in $K$ so that $\left\|g_{n+1}(\omega)-g_{n}(\omega)\right\| \geqq \delta^{\prime}$ for all $n$ and $\omega$. That is, $K$ contains a generalized $\delta^{\prime}$-tree.

Proof. (b) $\Rightarrow(\mathrm{a})$ : Let $\left(g_{n}\right)$ as in (b) and set $A=\left\{g_{n}(\omega): \omega \in \Omega\right.$, $n=0,1,2, \cdots\}$. Then $A$ has no $\delta^{\prime}$-approximate extreme points since in fact $A=G_{\dot{\delta}}(A)$.

$(\mathrm{a}) \Rightarrow(\mathrm{b})$ : Let $A$ as in (a); by Proposition 3.1, $A \subset \overline{G_{\hat{o}}(A)}$. Let $\delta^{\prime}<\delta^{\prime \prime}<\delta$ and let $\left(\varepsilon_{n}\right)$ satisfy (26). By the same technique as in the proof of Proposition 2.1, we may construct a generalized dyadic decomposition $\left(\mathscr{C}_{n}\right)$ and an $\infty$-quasi-martingale $\left(f_{n}\right)$ corresponding to $\left(\varepsilon_{j}\right)$ (with respect to $\left(\mathscr{A}_{n}\right)$ ) with $f_{n}$ valued in $A$ for all $n$ and satisfying (27). It follows as in the preceding argument that the martingale $\left(g_{n}\right)$ of Theorem 1.1 is valued in $\overline{\text { co }} A \subset K$ and satisfies (b) of 3.3, completing the proof.

Let us say that a closed bounded convex subset $K$ of a Banach 
space has the approximate Krein-Milman property (AKMP) if every nonempty subset of $K$ has an $\varepsilon$-strong extreme point for every $\varepsilon>0$; we say that a Banach space has the AKMP if its unit ball has this property. Suppose $K$ has the AKMP and $\varepsilon>0$. Is $K=$ $\overline{\text { co }} E_{\varepsilon}(K)$ ? We do not know the answer, except in special cases. The following result gives some information concerning this problem.

Proposition 3.4. Let $K$ be a closed bounded convex set and $\varepsilon>0$ so that $K \neq \overline{\mathrm{co}} E_{\varepsilon}(K)$. Then given $0<\delta<\varepsilon$ and $\kappa>0$, there exists a dyadic martingale $\left(g_{n}\right)$ valued in $K$ and a measurable set $B$ in our probability space with measure larger than $1-\kappa$ so that $\left\|g_{n+1}(\omega)-g_{n}(\omega)\right\| \geqq \delta$ for all $n$ and $\omega \in B$.

Proof. Let $f \in X^{*}$ with $\|f\|=1$ so that $f$ separates $\overline{c o} E_{\varepsilon}(K)$ from some point in $K$. (We can assume of course that $E_{\varepsilon}(K) \neq \varnothing$, or else 3.4 is trivial in virtue of Proposition 3.2.) Thus we may choose an $\eta>0$ so that $S(f, \eta)$ is disjoint from $\overline{c o} E_{\varepsilon}(K)$. Hence $S(f, \eta) \subset \overline{G_{\varepsilon}}(K)$ by Proposition 3.1. Thus if $k \in S(f, \eta)$ and $\gamma>0$, there exist $k_{1}, k_{2} \in K$ with $\left\|\left(k_{1}+k_{2}\right) / 2-k\right\|<\gamma$ and $\left\|k-k_{i}\right\| \geqq \varepsilon-\gamma$ for $i=1,2$.

Now let $k_{0} \in S(f, \eta)$ be such that

$$
f\left(k_{0}\right)>M-\eta \text { and } \frac{\eta-\left(M-f\left(k_{0}\right)\right)}{\eta}>1-\kappa,
$$

(where $M=\sup f(K)$ ).

Let $\delta<\delta^{\prime}<\varepsilon$, set $\tau=\eta-\left(M-f\left(k_{0}\right)\right)$, and let $\left(\varepsilon_{n}\right)$ be a sequence of positive numbers so that

$$
\delta_{0}=\sum_{j=0}^{\infty} \varepsilon_{j} \text { satisfies } \delta_{0} \leqq \frac{\delta^{\prime}-\delta}{2} \text { and } \frac{\tau-\delta_{0}}{\eta}>1-\kappa .
$$

By the technique of Proposition 3.2 and Lemma 2.4, we may choose a $k$-valued dyadic $\infty$-quasi-martingale $\left(h_{n}\right)$ corresponding to $\left(\varepsilon_{n}\right)$ so that for all $n$ and $\omega$,

$$
\begin{gathered}
h_{0} \equiv k_{0} \\
\text { if } f\left(h_{n}(\omega)\right) \geqq M-\eta,\left\|h_{n+1}(\omega)-h_{n}(\omega)\right\| \geqq \delta^{\prime}
\end{gathered}
$$

and

$$
\text { if } f\left(h_{n}(\omega)\right)<M-\eta \text {, then } h_{n+1}(\omega)=h_{n}(\omega) \text {. }
$$

Let $\left(g_{n}\right)$ be the dyadic martingale given by Theorem 1.1. Thus, $g_{n}$ is valued in $K$ and $\left\|g_{n}-h_{n}\right\|_{\infty} \leqq \delta_{n}$ for all $n$ (where $\delta_{n}=\sum_{j=n}^{\infty} \varepsilon_{j}$ ). Now for each $n$, let $B_{n}=\left[h_{n} \in S(f, \eta)\right]$. It follows by (32) that 
$B_{n} \supset B_{n+1}$ for all $n$; set $B=\bigcap_{n=1}^{\infty} B_{n}$. It now follows exactly by the argument given in the Remark following the proof of Lemma 2.4 that $P\left(B_{n}\right) \geqq\left(\tau-\delta_{n}-\delta_{0}\right) / \eta$ for all $n$. Hence

$$
P(B)=\lim _{n \rightarrow \infty} P\left(B_{n}\right) \geqq \frac{\tau-\delta_{0}}{\eta}>1-\kappa \text { by }(28) .
$$

Now let $\omega \in B$. Then

$$
\left\|h_{n+1}(\omega)-h_{n}(\omega)\right\| \geqq \delta^{\prime} \text { by }(31) .
$$

But

$$
\begin{aligned}
\left\|g_{n+1}(\omega)-g_{n}(\omega)\right\| & \geqq\left\|h_{n+1}(\omega)-h_{n}(\omega)\right\|-\left\|g_{n+1}-h_{n+1}\right\|_{\infty}-\left\|g_{n}-h_{n}\right\|_{\infty} \\
& \geqq \delta^{\prime}-\delta_{n+1}-\delta_{n} \geqq \delta^{\prime}-2 \delta_{0} \geqq \delta \text { by }(29),
\end{aligned}
$$

thus proving Proposition 3.4.

REMARKS. 1. Of course it follows immediately from 3.4 that $\left\|g_{n+1}-g_{n}\right\|_{1} \geqq \delta P(B)>0$ for all $n$. There are Banach spaces $X$ which fail the RNP, yet have the property that for all generalized dyadic uniformly bounded martingales $\left(g_{n}\right), \underline{\lim }_{n \rightarrow \infty}\left\|g_{n+1}-g_{n}\right\|_{1}=0$. (See [4].). By Proposition 3.4, such spaces $X$ satisfy: $K=\overline{\mathrm{co}} E_{\varepsilon}(K)$ for every $\varepsilon>0$ and closed bounded convex set $K$, and also the AKMP (by Proposition 3.3). It has been proved independently by J. Bourgain and somewhat later, by J. Elton, that the particular space $X$ constructed in [4] contains a closed bounded convex nonempty set $K$ with no extreme points. Thus the AKMP does not imply the KMP.

2. Let $X$ satisfy the AKMP and $K$ a convex body in $X$. That is, $K$ is closed convex with nonempty interior. Does $K$ have an extreme point?

3. Regard $X$ as being a subspace of $X^{* *}$ and let $K$ be a closed bounded convex subset of $X$; denote the weak*-closure of $K$ in $X^{* *}$ by $\tilde{K}$. Let us say that $k \in K$ is a weak* extreme point of $K$ if $k$ is an extreme point of $\widetilde{K}$. Now if $k \in K$ is a strong extreme point of $K$, then $k$ is a weak ${ }^{*}$ extreme point. Indeed, suppose this were false. Choose $F, G \in \widetilde{K}$ with $F \neq G$ and $k=(F+G) / 2$. Choose $\varphi \in X^{*}$ with $(F-G)(\varphi)=1$. Choose a net $\left(y_{\alpha}, z_{\alpha}\right)_{\alpha \in D}$ of points in $K$ with $y_{\alpha} \rightarrow F$ and $z_{\alpha} \rightarrow G$ weak*. Since then $y_{\alpha}-z_{\alpha} \rightarrow F-G$ weak*, $\varphi\left(y_{\alpha}-z_{\alpha}\right) \rightarrow 1$, hence we may assume without loss of generality that

$$
\varphi\left(y_{\alpha}-z_{\alpha}\right) \geqq \frac{1}{2} \text { for all } \alpha \in D \text {. }
$$

Set $\varepsilon=1 /(4\|\varphi\|)$; since $x$ is an $\varepsilon$-strong extreme point, we may 
choose $\delta>0$ so that for all $y, z \in K$,

$$
\left|\frac{y+z}{2}-x\right|<\delta \Longrightarrow\|y-z\|<2 \varepsilon
$$

Now $\left(y_{\alpha}+z_{\alpha}\right) / 2 \rightarrow x$ weakly. Hence we may choose $\alpha_{1}, \cdots, \alpha_{k}$ in $D$ and nonnegative scalars $\lambda_{1}, \cdots, \lambda_{k}$ with $\sum_{i=1}^{k} \lambda_{i}=1$ so that $\left\|\sum_{\imath=1}^{k} \lambda_{i}\left(\left(y_{\alpha_{i}}+z_{\alpha_{i}}\right) / 2\right)-x\right\|<\delta$. Set $y=\sum_{i=1}^{k} \lambda_{i} y_{\alpha_{i}}$ and $z=\sum_{i=1}^{k} \lambda_{i} z_{\alpha_{i}}$. Since $\|(y+z) / 2-x\|<\delta$, we have

$$
|\varphi(y-z)| \leqq\|\varphi\|\|y-z\|<2\|\varphi\| \frac{1}{4\|\varphi\|}=\frac{1}{2} \text { by }
$$

and the definition of $\varepsilon$. But

$$
\varphi(y-z)=\varphi\left(\sum_{i=1}^{k} \lambda_{i}\left(y_{\alpha_{i}}-z_{\alpha_{i}}\right)\right) \geqq \frac{1}{2} \text { by }(34),
$$

a contradiction.

Now the proof of a result of J. Bourgain (Corollary 4 of [3]) yields that $K$ has the RNP provided $L$ has a weak* extreme point for every closed bounded convex subset $L$ of $K$. Thus $K$ has the RNP provided every such $L$ has a strong extreme point.

4. Of course $k \in K$ is a strong extreme point of $K$ if and only if for every pair of sequences of points $\left(u_{n}\right)$ and $\left(v_{n}\right)$ of $K$ with $\left(u_{n}+v_{n}\right) / 2 \rightarrow k$ in norm, $\left\|u_{n}-v_{n}\right\| \rightarrow 0$. Evidently a locally uniformly convex Banach space has the property that every point of its unit sphere is a strong extreme point of its unit ball. Is a Banach space $X$ with this property locally uniformly convex?

\section{REFERENCES}

1. A. Bellow, Uniform amarts: a class of asymptotic martingales for which strong almost sure convergence obtains, Zeit. Wahrs. verw. Gebiete, 4 (1978), 177-191.

2. J. Bourgain, On dentability and the Bishop-Phelps property, Israel J. Math., 28 (1977), 265-271.

3. - A geometric characterization of the Radon-Nikodym property in Banach spaces, Compositio Math., 36 (1978), 3-6.

4. J. Bourgain and H. Rosenthal, Martingales valued in certain subspaces of $L^{1}$, Israel J. Math., 37 (1980), 54-75.

5. - Geometrical implications of certain finite dimensional decompositions, to appear, Bull. Soc. Math. Belg.

6. J. Diestel and J. J. Uhl, Jr., Vector Measures, Amer. Math. Soc. Mathematical Surveys 15, Providence, 1977.

7. G. A. Edgar and L. Sucheston, The Riesz decomposition for vector-valued amarts, Zeit. Wahrs. verw. Gebiete, 36 (1976), 85-92.

8. D. G. Larman and R. R. Phelps, Gateaux differentiability of convex functions on Banach spaces, J. London Math. Soc., 20 (1979), 115-127.

9. R. R. Phelps, Dentability and extreme points in Banach spaces, J. Functional Analysis, 17 (1974), 78-90. 
Received May 25, 1981. This research was partially supported by NSF grants MCS-8002132 and MCS-8002393.

The UnIVERSITY OF TEXAS

AUsTin, TX 78712 



\section{PACIFIC JOURNAL OF MATHEMATICS}

\section{EDITORS}

DONALD BABBITT (Managing Editor)

University of California

Los Angeles, California 90024

Hugo RossI

University of Utah

Salt Lake City, UT 84112

C. C. MOORE and ARTHUR Agus

University of California

Berkeley, CA 94720

\section{J. DUGUNDJI}

Department of Mathematics University of Southern California Los Angeles, California 90007

R. FinN and J. MILGRAM Stanford University Stanford, California 94305

\section{ASSOCIATE EDITORS}
R. ARNES
E. F. BeCKENBACH
B. H. Neumann
F. WOLF
K. YosHIDA

\section{SUPPORTING INSTITUTIONS}

UNIVERSITY OF ARIZONA

UNIVERSITY OF BRITISH COLUMBIA

CALIFORNIA INSTITUTE OF TECHNOLOGY

UNIVERSITY OF CALIFORNIA

MONTANA STATE UNIVERSITY

UNIVERSITY OF NEVADA, RENO

NEW MEXICO STATE UNIVERSITY

OREGON STATE UNIVERSITY
UNIVERSITY OF OREGON UNIVERSITY OF SOUTHERN CALIFORNIA STANFORD UNIVERSITY UNIVERSITY OF HAWAII UNIVERSITY OF TOKYO UNIVERSITY OF UTAH WASHINGTON STATE UNIVERSITY UNIVERSITY OF WASHINGTON 


\section{Pacific Journal of Mathematics}

Vol. 100, No. $1 \quad$ September, 1982

Charalambos D. Aliprantis, Owen Sidney Burkinshaw and M. Duhoux,

Compactness properties of abstract kernel operators $\ldots \ldots \ldots \ldots \ldots \ldots 1$

Roger C. Alperin, Locally compact groups acting on trees .............23

Robert F. Brown, Real homology of Lie group homomorphisms ......... 33

Karen Chase, Maximal groups in sandwich semigroups of binary relations . . 43

W. Wistar (William) Comfort and T. Soundararajan, Pseudocompact

group topologies and totally dense subgroups $\ldots \ldots \ldots \ldots \ldots \ldots \ldots 61$

M. Ferri and C. Gagliardi, Crystallisation moves $\ldots \ldots \ldots \ldots \ldots \ldots \ldots$

Kenneth R. Goodearl, Directly finite aleph-nought-continuous regular

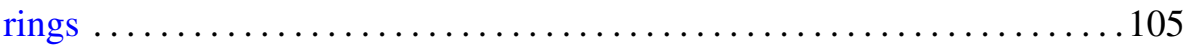

Edward Lewis Green, On the representation theory of rings in matrix

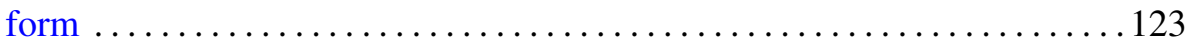

Walter Hengartner and Glenn E. Schober, Interpolation, continuation, and

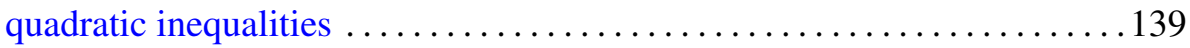

Kenneth Kunen and Haskell Paul Rosenthal, Martingale proofs of some geometrical results in Banach space theory $\ldots \ldots \ldots \ldots \ldots \ldots \ldots \ldots \ldots \ldots$

Brian William McEnnis, Shifts on indefinite inner product spaces. II . . . . . 177

Roman Pol, Note on the spaces $P(S)$ of regular probability measures whose topology is determined by countable subsets $\ldots \ldots \ldots \ldots \ldots \ldots \ldots \ldots 185$

Joan Manuel Verdera Melenchón, Finitely generated projective extensions

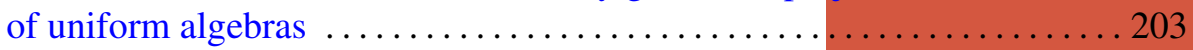

Cheng Ye You, Fixed point classes of a fiber map .................. 217 